\title{
Nonlinear travelling waves on a spiralling liquid jet
}

\author{
E. Părău, ${ }^{*}$ S. P. Decent, ${ }^{\dagger}$ and A. C. King \\ School of Mathematics and Statistics, The University of Birmingham, \\ Edgbaston, Birmingham, B15 2TT, UK \\ M. J. H. Simmons and D. C. Wong \\ Department of Chemical Engineering, The University of Birmingham, \\ Edgbaston, Birmingham, B15 2TT, UK
}

(Dated: 21 April 2006)

\begin{abstract}
We describe the nonlinear evolution of a travelling wave disturbance on a spiralling slender inviscid jet which emerges from a rotating orifice neglecting gravity. One-dimensional equations are derived using asymptotic methods and solved numerically. Some results are presented for this nonlinear theory which is rather different from previous linear theories, showing the influence of surface tension and rotation on the breakup of the jets into droplets. Comparison with the experimental results shows good qualitative agreement.
\end{abstract}

PACS numbers: 47.27.Wg 


\section{INTRODUCTION}

In this paper we analyse the growth of unstable travelling wave disturbances on a slender, inviscid curved jet which emerges from a rapidly rotating container. This problem arises in the manufacture of fertiliser and magnesium pellets using the so-called "prilling" process $\left(\right.$ Andersen $\left.^{1}\right)$. Here, molten urea or magnesium flows into a rapidly rotating cylindrical container which has many thousands of small holes on the curved surface. The container rotates about its axis so that the liquid is forced out of the holes in the form of slender jets which breakup into droplets due to a surface tension driven instability. These droplets then fall and solidify to form pellets. The equations of motion for such a jet in a rotating reference frame were given by Wallwork et $a .^{2}$ They determined the trajectory of the jet using asymptotic methods, and the linear stability using a multiple scales approach. Gravity was included in Decent et al. ${ }^{3}$

Nonlinear one-dimensional models for axisymmetric straight jets have been developed, by assuming a periodic disturbance along the infinite jet, by many authors (see Lee, ${ }^{4}$ Mansour and Lundgren, ${ }^{5}$ Schulkes, ${ }^{6}$ Papageorgiou and Orellana $\left.{ }^{7}\right)$. The presence of the orifice has also been included, first by Keller et $a l^{33}$, and in recent jet simulations, which consider the jet having a finite length (see Eggers and Dupont, ${ }^{8}$ Hilbing and Heister, ${ }^{9}$ Cheong ${ }^{10}$ ). An extensive review of the work on straight axisymmetric liquid jets is given by Eggers ${ }^{11}$ and Vanden-Broeck. $^{12}$

Curved liquid jets and sheets have been studied before by Weber ${ }^{13}$ Vanden-Broeck and Keller $^{14}$, Dias and Vanden-Broeck ${ }^{15}$, Finnicum et al. ${ }^{16}$, Cummings and Howell ${ }^{17}$, Entov and Yarin $^{18}$, Yarin ${ }^{19}$, Reneker it et al. ${ }^{20}$, Yarin et $a .^{21}$ Hohman et $a .^{22}$ and others. Here we extend these works and Wallwork et al. ${ }^{2}$ to develop a long wavelength nonlinear theory for slender inviscid jets subjected to rotational forces. An asymptotic analysis is performed in Section II, in order to obtain a coupled partial differential equation system for travelling waves on a curved jet with a steady trajectory in the rotating frame moving with the container. In Section III a numerical method is presented to solve the leading order equations. Typical results, showing the influence of various parameters, are presented in Section IV and a comparison with experimental results shows good qualitative agreement. In Section $\mathrm{V}$ a further system of equations are produced which allow an unsteady trajectory and which describe waves with a wavelength which is very long compared to those discussed in Sec- 
tions II to IV. These equations are also solved numerically and the results discussed. Some conclusions are made in Section VI.

\section{ONE-DIMENSIONAL MODEL FOR A NONLINEAR DISTURBANCE ON A CURVED JET}

An inviscid liquid jet leaves a small orifice of radius $a$ situated on the curved face of a circular cylindrical container of radius $s_{0}$, rotating at a constant rate $\Omega$ about the axis of the cylinder. We work in a rotating reference frame in which the orifice is fixed and we use a curvilinear coordinate system $(s, n, \phi)$ where $s$ is the arclength along the centreline of the jet, and $(n, \phi)$ are plane polar coordinates in any cross-section of the jet. These coordinates have unit vectors denoted by $\mathbf{e}_{s}, \mathbf{e}_{n}$ and $\mathbf{e}_{\phi}$ respectively.

To simplify our analysis we neglect gravity as in Wallwork et al. ${ }^{2}$ This can be justified by the fact that the jets do not fall significantly under gravity before they breakup into droplets in prilling since $s_{0}^{2} \Omega>>g$, where $g$ is the acceleration due to gravity. The centreline of the jet in Cartesian coordinates is described by $(X, 0, Z)$ with the origin at the center of the orifice. The $x$-axis is directed normal to the surface of the container in the initial direction of the jet with the $z$-axis lying in the plane of the centreline of the jet. Here $X$ and $Z$ are functions of arclength $s$ and time $t$.

The velocity of the fluid is $\underline{u}=u \mathbf{e}_{s}+v \mathbf{e}_{n}+w \mathbf{e}_{\phi}$. We consider flow without azimuthal rotation $(w=0)$ and we use the scalings, as in Wallwork et al. ${ }^{2}$,

$$
\begin{aligned}
& \hat{u}=\frac{u}{U}, \hat{v}=\frac{v}{U}, \hat{p}=\frac{p}{\rho U^{2}}, \hat{n}=\frac{n}{a}, \epsilon=\frac{a}{s_{0}} \\
& \hat{R}=\frac{R}{a}, \hat{s}=\frac{s}{s_{0}}, \hat{t}=\frac{t U}{s_{0}}, \hat{X}=\frac{X}{s_{0}}, \hat{Z}=\frac{Z}{s_{0}}
\end{aligned}
$$

where $U$ is the exit speed of the jet in the rotating frame, $R=R(s, t)$ is the radius of the jet, $\rho$ is the liquid's density, $p$ is the pressure, $u$ and $v$ are the tangential and radial velocity components relative to the centreline of the jet and $\epsilon$ the aspect ratio of the jet. The hats denote dimensionless quantities. If the hats are dropped then this gives the equations of motion ((2.3)-(2.9) in Wallwork et al. $\left.{ }^{2}\right)$.

We now suppose that the liquid jet is slender and $\epsilon$ is small. The lengthscale over which the centreline curves is $\hat{s}=O(1)$ (so long as the Rossby number $R b=U /\left(s_{0} \Omega\right)=O(1)$ : see Wallwork et $a .^{2}$ ), but the travelling wave perturbations to the jet which are observed 
in experiments and which cause the breakup of the jet into droplets have a much smaller wavelength. We suppose that we have a perturbation to the jet with initial (dimensional) wavelength $\lambda$, where $\lambda<<s_{0}$. A sketch of the typical flow is shown in Fig. 1. We introduce the short dimensionless lengthscale $\bar{s}=\frac{s}{\lambda}=\frac{\gamma}{\epsilon} \hat{s}$, where $\gamma=a / \lambda$. We also introduce a dimensionless timescale for these perturbations $\bar{t}=\frac{\gamma}{\epsilon} \hat{t}$. Usually, $\gamma<<1$ (a long-wavelength hypothesis) and $\epsilon<<\gamma$, so that $s_{0}>>\lambda>>a$. This means that unstable travelling wave disturbances are usually much longer than the jet radius but much shorter than the radius of the container, as observed in our experiments. ${ }^{2}$

Using these new scales, we can rewrite the equations given by Wallwork et $a .^{2}$ and (dropping hats, but not overbars) we obtain the equation for the conservation of mass

$$
\begin{aligned}
\epsilon n \frac{\partial u}{\partial s}+\gamma n \frac{\partial u}{\partial \bar{s}} & +\left(1+\epsilon n \cos \phi\left(X_{s} Z_{s s}-X_{s s} Z_{s}\right)\right)\left(v+n \frac{\partial v}{\partial n}\right)+ \\
& +\epsilon n \cos \phi\left(X_{s} Z_{s s}-X_{s s} Z_{s}\right) v=0
\end{aligned}
$$

Euler's equations

$$
\begin{gathered}
\left(1+\epsilon n \cos \phi\left(X_{s} Z_{s s}-X_{s s} Z_{s}\right)\right)\left(\epsilon \frac{\partial u}{\partial t}+\gamma \frac{\partial u}{\partial \bar{t}}+\epsilon v \cos \phi\left(Z_{s t} X_{s}-X_{s t} Z_{s}\right)+v \frac{\partial u}{\partial n}\right) \\
+\epsilon u \frac{\partial u}{\partial s}+\gamma u \frac{\partial u}{\partial \bar{s}}+\epsilon u\left(X_{s} Z_{s s}-X_{s s} Z_{s}\right) v \cos \phi=-\epsilon \frac{\partial p}{\partial s}-\gamma \frac{\partial p}{\partial \bar{s}}+ \\
+\left(2 \frac{\epsilon}{R b} v \cos \phi+\frac{\epsilon}{R b^{2}}\left((X+1) X_{s}+Z Z_{s}\right)\right)\left(1+\epsilon n \cos \phi\left(X_{s} Z_{s s}-X_{s s} Z_{s}\right)\right)
\end{gathered}
$$

and

$$
\begin{gathered}
\left(1+\epsilon n \cos \phi\left(X_{s} Z_{s s}-X_{s s} Z_{s}\right)\right)\left(\epsilon \frac{\partial v}{\partial t}+\gamma \frac{\partial v}{\partial \bar{t}}+\epsilon u \cos \phi\left(X_{s t} Z_{s}-Z_{s t} X_{s}\right)+v \frac{\partial v}{\partial n}\right) \\
+\epsilon u \frac{\partial v}{\partial s}+\gamma u \frac{\partial v}{\partial \bar{s}}-\epsilon \cos \phi\left(X_{s} Z_{s s}-X_{s s} Z_{s}\right) u^{2}=\left(-\frac{\partial p}{\partial n}-2 \frac{\epsilon}{R b} u \cos \phi\right. \\
\left.+\frac{\epsilon}{R b^{2}} \cos \phi\left((X+1) Z_{s}-Z X_{s}+\epsilon n \cos \phi\right)\right)\left(1+\epsilon n \cos \phi\left(X_{s} Z_{s s}-X_{s s} Z_{s}\right)\right) \\
\left(1+\epsilon n \cos \phi\left(X_{s} Z_{s s}-X_{s s} Z_{s}\right)\right)\left(\epsilon u \sin \phi\left(Z_{s t} X_{s}-X_{s t} Z_{s}\right)\right)+ \\
+\epsilon \sin \phi\left(X_{s} Z_{s s}-X_{s s} Z_{s}\right) u^{2}=\left(-\frac{1}{n} \frac{\partial p}{\partial \phi}+2 \frac{\epsilon}{R b} u \sin \phi+\right. \\
\left.+\frac{\epsilon}{R b^{2}} \sin \phi\left(Z X_{s}-(X+1) Z_{s}-\epsilon n \cos \phi\right)\right)\left(1+\epsilon n \cos \phi\left(X_{s} Z_{s s}-X_{s s} Z_{s}\right)\right)
\end{gathered}
$$

the kinematic condition

$$
\left(1+\epsilon n \cos \phi\left(X_{s} Z_{s s}-X_{s s} Z_{s}\right)\right)\left(\epsilon \frac{\partial R}{\partial t}+\gamma \frac{\partial R}{\partial \bar{t}}+\left(Z_{s} X_{t}-X_{s} Z_{t}\right) \cos \phi-\right.
$$




$$
-v)+\epsilon u \frac{\partial R}{\partial s}+\gamma u \frac{\partial R}{\partial \bar{s}}=0 \quad \text { on } n=R
$$

the dynamic condition

$$
p=\frac{\kappa}{W e} \quad \text { on } n=R
$$

where

$$
\begin{gathered}
\kappa=\frac{1}{h}\left(\epsilon^{2} \frac{\partial\left(-\frac{1}{h E} \frac{\partial R}{\partial s}\right)}{\partial s}+\gamma \epsilon\left(\frac{\partial\left(-\frac{1}{h E} \frac{\partial R}{\partial s}\right)}{\partial \bar{s}}+\frac{\partial\left(-\frac{1}{h E} \frac{\partial R}{\partial \bar{s}}\right)}{\partial s}\right)+\right. \\
\left.+\gamma^{2} \frac{\partial\left(-\frac{1}{h E} \frac{\partial R}{\partial \bar{s}}\right)}{\partial \bar{s}}+\frac{1}{n} \frac{\partial\left(\frac{n h}{E}\right)}{\partial n}+\frac{\partial}{\partial \phi}\left(-\frac{h}{n^{2} E} \frac{\partial R}{\partial \phi}\right)\right) \\
h=1+\epsilon n \cos \phi\left(X_{s} Z_{s s}-X_{s s} Z_{s}\right) \text { and } \\
E=\left(1+\frac{\epsilon^{2}}{h^{2}}\left(\frac{\partial R}{\partial s}\right)^{2}+2 \frac{\epsilon \gamma}{h^{2}} \frac{\partial R}{\partial s} \frac{\partial R}{\partial \bar{s}}+\frac{\gamma^{2}}{h^{2}}\left(\frac{\partial R}{\partial \bar{s}}\right)^{2}+\frac{1}{n^{2}}\left(\frac{\partial R}{\partial \phi}\right)^{2}\right)^{1 / 2}
\end{gathered}
$$

the arclength condition

$$
X_{s}^{2}+Z_{s}^{2}=1
$$

and

$$
v=0 \quad \text { on } \quad n=0 .
$$

The non-dimensional parameters here are the Weber number $W e=\rho U^{2} a / \sigma$ and the Rossby number $R b=U /\left(s_{0} \Omega\right)$. They describe the relative importance of the forces due to surface tension and rotation relative to inertia, respectively. We should note a small correction on the left-hand side of the equation (5): the term $\frac{v}{n}\left(Z_{s} X_{t}-Z_{t} X_{s}\right)$ which appears by mistake in Wallwork et $a .^{2}$ is removed here.

In Wallwork et $a .^{2}{ }^{2}$ a multiple scales analysis was used to determine the linear instability of the steady basic state by perturbing this steady curved liquid jet by linear travelling waves. In these calculations it was shown that the periodicity of these linear waves is associated with a short length and timescale, while the wavenumber $k$, frequency $\omega$ and amplitude of the mode $\delta$ all depend on the long lengthscale $s$ and timescale $t$. When a weakly nonlinear calculation is carried out for large $W e$, a Landau-type equation is found to describe the variation of the amplitude of this wave on the long lengthscale $s$, associated with cubic secularity in the wave amplitude $\delta$, so long as $\gamma^{2} \sim \epsilon{ }^{23}$. We can think of a travelling wave being initially linear near the orifice, then weakly nonlinear and finally strongly nonlinear as it travels upstream. (This is a distinguished limit arising in the equations of motion. For example, this corresponds to a balance between a linear term in the (long) derivative of 
the amplitude of the weakly nonlinear mode $\delta_{s}$ which arises from the $\epsilon p_{s}$ term in (3) with a nonlinear term $|\delta|^{2} \delta$ resulting from an interaction between 1st and 2nd harmonic wave modes in the $v u_{n}$ term in (3). If the steady basic state is $O(1)$ then the weakly nonlinear 1 st harmonic mode has amplitude of order of the size $\gamma$ since it is associated with the next largest terms such as $u_{1}$ and $p_{1}$ in the expansion that follows, while the 2 nd harmonic will have size of order $\gamma^{2}$ since these terms result from quadratic interaction between 1st harmonics etc. Therefore, this gives a balance between terms of size $\epsilon \gamma$ and $\gamma^{3}$ respectively, resulting in the distinguished limit $\gamma^{2} \sim \epsilon$. Such Landau-type equations are described in detail in $\mathrm{Craik}^{24}$, amongst others.) An analogous result was found before by Schulkes for a straight jet ${ }^{6}$ and by Yarin $^{19}$ for liquid threads. Here we wish to use a long wavelength theory to generalise these multiple scales linear and weakly nonlinear instability results of Wallwork el. ${ }^{2,13}$ onto a strongly nonlinear setting (so that the leading-order terms in the expansion may also contain wave-like behaviour), and so we continue with the limit $\gamma^{2} \sim \epsilon$ here. The consequence of this scaling is that it would in principal be possible to continue our asymptotic expansion to higher orders in a consistent way, so that linear and weakly nonlinear theories would be a subset of the work presented here.

In fact there is experimental evidence to support this distinguished limit. In all laboratory experiments and industrial set-ups of this liquid jet configuration ${ }^{1,2,13,25,26}$, the aspect ratio of the jet $\epsilon$ is always of the order of $10^{-2}$ for low viscosity liquids. We have previously carried out several experiments using different rotating containers, with $a$ and $s_{0}$ of various sizes. In all cases we have needed to use an $\epsilon$ approximately of this order to form a coherent nonturbulent liquid jet. If $\epsilon$ is an order of magnitude larger, then experimental results suggest that the jet is not particularly slender, and possibly even turbulent, and hence outside the range of theories considered here ${ }^{13,25}$. When $\epsilon$ is an order of magnitude smaller than $10^{-2}$ then it is very difficult is achieve a liquid jet with an $O(1)$ break-up length. This is because reducing the size of the orifice also reduces the Weber number $W e$ which in turn reduces the length of the jet (as for straight jets too), and direct atomisation of the jet at the orifice is usually observed. (It is possible to decrease the size of the orifice $a$ and keep the Weber number $W e$ constant by increasing the exit speed of the jet at the orifice $U$. However, the practical engineering of this usually also results in a larger disturbance at the orifice $\delta$ which then decreases the break-up length of the jet.) When considering typical sizes of experimentally observed wavelengths $\lambda$, we note from Wallwork at al. ${ }^{2}$ that the unstable 
mode has wavenumber of the order $k=0.697 / a$, with $\lambda=2 \pi / k$, so that $\gamma=a / \lambda$ is of the order of $10^{-1}$ for a low viscosity liquid. We should mention here that the value of the wavenumber $k=0.697 / a$ was first found by Rayleigh ${ }^{27}$ for straight jets. This agrees with measurements of wavelengths from these experiments. Hence we see that $\epsilon \approx \gamma^{2}$ in the experiments. It is more difficult to achieve detailed measurements in any industrial setting, but the evidence available also supports this limit. (However, it should be noted that these arguments do not hold for a very viscous jet: this is the subject of current work where a different limit must be used instead.) We additionally consider instead the limit $\epsilon \sim \gamma$ here in Section V.

We write $\epsilon=K \gamma^{2}$ where $K$ in an $O(1)$ constant, and use the asymptotic slender jet series

$$
\begin{gathered}
u=u_{0}(s, \bar{s}, t, \bar{t})+\gamma u_{1}(s, \bar{s}, n, \phi, t, \bar{t})+\gamma^{2} u_{2}(s, \bar{s}, n, \phi, t, \bar{t})+\cdots, \\
v=\gamma v_{1}(s, \bar{s}, n, \phi, t, \bar{t})+\gamma^{2} v_{2}(s, \bar{s}, n, \phi, t, \bar{t})+\cdots, \\
p=p_{0}(s, \bar{s}, n, \phi, t, \bar{t})+\gamma p_{1}(s, \bar{s}, n, \phi, t, \bar{t})+\gamma^{2} p_{2}(s, \bar{s}, n, \phi, t, \bar{t})+\cdots, \\
R=R_{0}(s, \bar{s}, t, \bar{t})+\gamma R_{1}(s, \bar{s}, n, \phi, t, \bar{t})+\gamma^{2} R_{2}(s, \bar{s}, n, \phi, t, \bar{t})+\cdots, \\
X(s, t)=X_{0}(s)+\gamma^{2} X_{1}(s, t)+\cdots, \text { and } Z(s, t)=Z_{0}(s)+\gamma^{2} Z_{1}(s, t)+\cdots
\end{gathered}
$$

We assume that the position of centreline is not affected by the small perturbations, and at leading order, is not time-dependent.

As an observation, it is possible to have $X_{0}$ and $Z_{0}$ as functions of time. This would give rise to an extra equation found from the kinematic condition, at lower order than the main equation (see below). This extra equation would describe any temporal translation of the centre line of the jet. This has been observed in our viscous experiments when the centreline is sometimes displaced during recoil after droplet detachment for a high viscosity liquid such as glycerol, ${ }^{25}$ but not for small viscosity liquids. This extra equation obtained from the kinematic condition would also need to contain leading-order contributions from expansions in $v$ and $w$ if $X_{0}$ and $Z_{0}$ were to be allowed to be time-dependent. In that case the kinematic condition at leading-order would become

$$
\left(Z_{0 s} X_{0 t}-X_{0 t} Z_{0 s}\right) \cos \phi=v_{0}
$$

where the expansion in $v$ would additionally have to contain the leading-order term $v_{0}(s, \bar{s}, t, \bar{t}, \phi)$ and $w$ would contain the leading-order term $w_{0}(s, \bar{s}, t, \bar{t}, \phi)$. These terms in $v_{0}$ and $w_{0}$ correspond to velocity components which translate the centreline in the plane $y=0$. 
In this section we assume $v_{0}=w_{0}=0$ and $X_{0 t}=Z_{0 t}=0$. However, we later produce an equation (12) which will give a measure of the error of this assumption. We find in our numerical simulation that this error is always small except possibly at the time and location of breakup, at which our slender jet theory breaks down in any case. This agrees with our experimental observations ${ }^{2}$ for low viscosity liquids. This is discussed further in section IV and in section VI. In section V we will generalise this calculation to produce a model for which $X_{0 t}$ and $Z_{0 t}$ are non-zero.

From now on, we write $X_{0}$ and $Z_{0}$ as $X$ and $Z$ respectively, for simplicity.

From the continuity equation we obtain

$$
v_{1}=-\frac{n}{2} u_{0 \bar{s}}, \quad \text { and } v_{2}=-\frac{n}{2}\left(u_{1 \bar{s}}+K u_{0 s}\right) .
$$

By using the radial and azimuthal equations we can show

$$
p=p_{0}(s, \bar{s}, t, \bar{t})+\gamma p_{1}(s, \bar{s}, t, \bar{t})+\gamma^{2} p_{2}(s, \bar{s}, n, \phi, t, \bar{t})
$$

where

$$
\begin{gathered}
p_{2}(s, \bar{s}, n, \phi, t, \bar{t})=K n \cos \phi\left(u_{0}^{2}\left(X_{s} Z_{s s}-X_{s s} Z_{s}\right)-2 \frac{u_{0}}{R b}+\right. \\
\left.+\frac{1}{R b^{2}}\left((X+1) Z_{s}-Z X_{s}\right)\right)+\frac{n^{2}}{8}\left(2 u_{0 \bar{s} \bar{t}}-u_{0 \bar{s}}^{2}+2 u_{0} u_{0 \bar{s} \bar{s}}\right)+\bar{p}_{2}(s, \bar{s}, t, \bar{t}) .
\end{gathered}
$$

On the other hand, from the pressure condition at the surface, we have

$$
\begin{gathered}
p_{\mid n=R}=\frac{1}{W e}\left(\frac{1}{R_{0}}+\gamma\left(-\frac{R_{1}+R_{1 \phi \phi}}{R_{0}^{2}}\right)+\gamma^{2} \frac{1}{2}\left(-2 R_{0 \bar{s} \bar{s}}+\right.\right. \\
\left.\left.+\frac{4 R_{1 \phi \phi} R_{1}+R_{1 \phi}^{2}+2 R_{1}^{2}}{R_{0}^{3}}-2 \frac{R_{2 \phi \phi}+R_{2}}{R_{0}^{2}}-\frac{R_{0 \bar{s}}^{2}}{R_{0}}\right)+K \cos \phi\left(X_{s} Z_{s s}-X_{s s} Z_{s}\right)\right) .
\end{gathered}
$$

Now, if we compare the last expressions for pressures we obtain

$O(1): \quad p_{0}(s, \bar{s}, t, \bar{t})=\frac{1}{W e R_{0}}$,

$O(\gamma): \quad p_{1}(s, \bar{s}, t, \bar{t})=-\frac{R_{1}+R_{1 \phi \phi}}{W e R_{0}^{2}}$

and

$$
\begin{aligned}
& O\left(\gamma^{2}\right): f_{0}(s, \bar{s}, t, \bar{t})+K R_{0} \cos \phi\left(u_{0}^{2}\left(X_{s} Z_{s s}-X_{s s} Z_{s}\right)-\frac{2 u_{0}}{R b}+\right. \\
& \left.+\frac{1}{R b^{2}}\left((X+1) Z_{s}-Z X_{s}\right)\right)=-\frac{R_{2}+R_{2 \phi \phi}}{W e R_{0}^{2}}+\frac{4 R_{1 \phi \phi} R_{1}+R_{1 \phi}^{2}+2 R_{1}^{2}}{W e R_{0}^{3}}+ \\
& +K \frac{1}{W e} \cos \phi\left(X_{s} Z_{s s}-X_{s s} Z_{s}\right),
\end{aligned}
$$

where

$$
f_{0}(s, \bar{s}, t, \bar{t})=\frac{R_{0}^{2}}{8}\left(2 u_{0 \bar{s} \bar{t}}-u_{0 \bar{s}}^{2}+2 u_{0} u_{0 \bar{s} \bar{s}}\right)+\bar{p}_{2}(s, \bar{s}, t, \bar{t})+\frac{R_{0 \bar{s} \bar{s}}+\frac{R_{0 \bar{s}}^{2}}{2 R_{0}}}{W e} .
$$


From the $O(\gamma)$ relation we obtain $R_{1}=g_{1}(s, \bar{s}, t, \bar{t})+g_{2}(s, \bar{s}, t, \bar{t}) \cos \phi+g_{3}(s, \bar{s}, t, \bar{t}) \sin \phi$. Then the term $\frac{4 R_{1 \phi \phi} R_{1}+R_{1 \phi}^{2}+2 R_{1}^{2}}{W e R_{0}^{3}}$ from the $O\left(\gamma^{2}\right)$ relation becomes, after some calculations, $h_{1}(s, \bar{s}, t, \bar{t})+h_{2}(s, \bar{s}, t, \bar{t}) \cos 2 \phi+h_{3}(s, \bar{s}, t, \bar{t}) \sin 2 \phi$, where $h_{i}$ depend on $g_{i}$. As we shall see, their expressions are not important, so we will not give them explicitly.

At the $O\left(\gamma^{2}\right)$, we obtain

$$
R_{2 \phi \phi}+R_{2}=f_{1} \cos \phi+f_{2}+f_{3} \cos 2 \phi+f_{4} \sin 2 \phi
$$

where

$$
\begin{gathered}
f_{1}(s, \bar{s}, t, \bar{t})=K R_{0}^{2}\left(W e R _ { 0 } \left(u_{0}^{2}\left(X_{s} Z_{s s}-X_{s s} Z_{s}\right)-\frac{2 u_{0}}{R b}+\right.\right. \\
\left.\left.+\frac{1}{R b^{2}}\left((X+1) Z_{s}-Z X_{s}\right)\right)-\left(X_{s} Z_{s s}-X_{s s} Z_{s}\right)\right)
\end{gathered}
$$

and $f_{2}, f_{3}, f_{4}$ can be easily calculated from the previous relations. To obtain a solvability condition for $R_{2}$, we want $R_{2} / R_{0}$ to be bounded, so the inhomogenous part of the equation for $R_{2}$ should be orthogonal to the solution of the homogenous problem (see Nayfeh ${ }^{28}$ ). So we multiply (10) by $\hat{R}$ where $\hat{R}$ satisfies the homogenous problem

$$
\hat{R}_{\phi \phi}+\hat{R}=0
$$

and we integrate it

$$
\int_{0}^{2 \pi}\left(R_{2 \phi \phi}+R_{2}\right) \hat{R} d \phi=\int_{0}^{2 \pi}\left(f_{1} \cos \phi+f_{2}+f_{3} \cos 2 \phi+f_{4} \sin 2 \phi\right) \hat{R} d \phi .
$$

The left-hand side of the equation can be integrated by parts and we obtain that it is zero. When we integrate the right-hand side, using the fact that $\hat{R}=\alpha \cos \phi+\beta \sin \phi$, it can be shown that the only term which is not zero is $\int_{0}^{2 \pi} \cos \phi f_{1} \hat{R} d \phi=\alpha \pi f_{1}$. Hence the solvability condition is $f_{1}=0$ which gives

$$
u_{0}^{2}\left(X_{s} Z_{s s}-X_{s s} Z_{s}\right)-\frac{2 u_{0}}{R b}+\frac{1}{R b^{2}}\left((X+1) Z_{s}-Z X_{s}\right)-\frac{\left(X_{s} Z_{s s}-X_{s s} Z_{s}\right)}{W e R_{0}}=0
$$

Now the kinematic condition and the axial momentum equations at order $O(\gamma)$ gives us the system

$$
\begin{gathered}
R_{0 \bar{t}}+\frac{R_{0}}{2} u_{0 \bar{s}}+u_{0} R_{0 \bar{s}}=0 \\
u_{0 \bar{t}}+u_{0} u_{0 \bar{s}}=-p_{0 \bar{s}}
\end{gathered}
$$


where $p_{0}=\frac{1}{W e} \frac{1}{R_{0}}$. These equations (13) and (14) were also obtained before for straight axisymmetric jets by other authors. ${ }^{6,7,11}$ However, the rotation of the container $\Omega$ enters the formulation via the initial conditions for the above system of equations, and so this system is fundamentally different to those for straight jets. We shall see in the following sections how the solutions to these equations depend critically upon the rotation of the container, and how therefore the solution to this system of equations is different to the solution of the straight jet equations. The final equation required to close the system is

$$
X_{s}^{2}+Z_{s}^{2}=1
$$

The system (12)-(15) will be solved numerically. A comparison between these equations and the ones for a bending jet, derived using a different method, by Entov and Yarin ${ }^{18}$ and Yarin $^{19}$ is presented in the Appendix.

It is worth noting that, if we suppose that there is no disturbance to the jet (so the derivatives on $\bar{s}$ and $\bar{t}$ are vanishing) and we search only for steady-state solutions, we obtain at leading order the same equations as Wallwork et al. ${ }^{2}$ for a steady jet.

Equations (13) and (14) form a closed set of equations for $u_{0}$ and $R_{0}$. We will solve these equations to determine $u_{0}$ and $R_{0}$, starting with the steady solutions found in Wallwork et $a l^{2}$ as initial conditions. We will then examine (12) and (15) later. It is found that (12) and (15) are satisfied until break up when our theory fails. The steady solutions for $X$ and $Z$ are found from (12) and (15).

\section{NUMERICAL METHOD}

The nonlinear system to be solved is (13)-(14). We can perform a linear (temporal) stability analysis of the steady solutions $u_{0}(s)$ and $R_{0}(s)$. If we perturb the steady solutions using

$$
u_{0}=u_{0}(s)+\delta \tilde{u}(s, \bar{s}, \bar{t}), R_{0}=R_{0}(s)+\delta \tilde{R}(s, \bar{s}, \bar{t})
$$

where $\delta$ is small, the corresponding linearized equations at order $\delta$ are

$$
\tilde{R}_{\bar{t}}+\frac{R_{0}}{2} \tilde{u}_{\bar{s}}+u_{0} \tilde{R}_{\bar{s}}=0
$$

and

$$
\tilde{u}_{\bar{t}}+u_{0} \tilde{u}_{\bar{s}}=\frac{1}{W e} \frac{\tilde{R}_{\bar{s}}}{R_{0}^{2}}
$$


We will look now for solutions of the form

$$
\tilde{u}(s, \bar{s}, \bar{t})=\hat{u}(s) e^{i k \bar{s}+\lambda \bar{t}}, \tilde{R}(s, \bar{s}, \bar{t})=\hat{R}(s) e^{i k \bar{s}+\lambda \bar{t}}
$$

where $k=k(s)$ and $\lambda=\lambda(s)$, and we obtain the relation

$$
\lambda=-i k u_{0} \pm \frac{k}{\sqrt{2 W e R_{0}}} .
$$

This indicates instability to modes of all wavenumbers $k$ and not just for $0<k<1 /\left(\gamma R_{0}\right)$, as in the case of Wallwork et al. ${ }^{2}$ who performed the equivalent linear stability analysis of steady solutions. An explanation of this is that our analysis in section II is a long-wavelength analysis, so that to obtain the above solutions from Wallwork et al. ${ }^{2}$ it is necessary to take the limit $k \rightarrow 0$.

In the review of Eggers $^{11}$ [p.890] for axisymmetric jets, this instability to very short wavelength perturbations (i.e. the existence of an instability as $k \rightarrow \infty$ ) makes the system unsuitable for numerical methods. One way to inhibit this phenomenon is to replace the leading order pressure $p_{0}=\frac{1}{W e} \frac{1}{R_{0}}$ by the expression for the full curvature

$$
p=\frac{1}{W e}\left[\frac{1}{R_{0}\left(1+\gamma^{2} R_{0_{\bar{s}}}^{2}\right)^{1 / 2}}-\frac{\gamma^{2} R_{0_{\bar{s} \bar{s}}}}{\left(1+\gamma^{2} R_{0_{\bar{s}}}^{2}\right)^{3 / 2}}\right] .
$$

The first to use this method was Lee, ${ }^{4}$ and it was applied with good results by many other authors (see for example Papageorgiou \& Orellana, ${ }^{7}$ Eggers and Duppont ${ }^{8}$ ). It is also worth noting that Entov and Yarin ${ }^{18}$ and Yarin ${ }^{19}$ have obtained the pressure in the form (19) in their derivation of the quasi-one-dimensional equations for bending jets, using physical arguments. If the above linear stability analysis is repeated for this expression of the pressure, we instead obtain the physically correct result of instability for $0<k<1 /\left(\gamma R_{0}\right)$.

A suitable numerical method can be obtained by modifying the method of Zhu et al. ${ }^{29}$, which was used to solve numerically a liquid jet falling into a liquid pool.

We now rescale the variables in this problem as $T=\frac{t U}{a}, S=\frac{s}{a}$ (here $t$ and $s$ are the initial dimensional parameters) and, after changing the notations $R_{0} \rightarrow R$ and $u_{0} \rightarrow u$, the system to be solved is

$$
\begin{gathered}
R_{T}+\frac{R}{2} u_{S}+u R_{S}=0 \\
u_{T}+u u_{S}=-\frac{1}{W e} \frac{\partial}{\partial S}\left[\frac{1}{R\left(1+R_{S}^{2}\right)^{1 / 2}}-\frac{R_{S S}}{\left(1+R_{S}^{2}\right)^{3 / 2}}\right] .
\end{gathered}
$$


We preferred to use this scaling based on initial radius and not the one based in the wavelength $\lambda$ for numerical reasons. A similar rescaling, but for a different problem was used in Li and Sclavounos, ${ }^{30}$ where the model equations for three-dimensional solitary waves in shallow water were deduced using a scaling based on wavelength and the numerical computations were performed using another scaling, based on water depth.

This system is solved using a finite-difference method. The spatial grid is fixed and uniform. The time integration method is based on a explicit scheme, using the Lax-Wendroff method.

If we denote $A=R^{2}$ the system (20)-(21) can be rewritten as a hyperbolic system:

$$
\begin{gathered}
A_{T}+(A u)_{S}=0 \\
u_{T}+\left(\frac{u^{2}}{2}\right)_{S}=-\frac{1}{W e} \frac{\partial}{\partial S} \frac{4\left(2 A+A_{S}^{2}-A_{S S}\right)}{\left(4 A+A_{S}^{2}\right)^{3 / 2}} .
\end{gathered}
$$

We solve the system (22)-(23) for $S \in[0, l]$ where $l$ is the length of the (numerical) jet. We define $n$ equally spaced points $S_{0}=0$ (the nozzle), $S_{i}=S_{0}+i \times d S, i=1, n$ where $d S=l / n$ is the spatial grid interval. The magnitude of the time step is denoted by $d T$.

The system was solved numerically by the Lax-Wendroff two-stage scheme (see e.g. Ref. 31, p.835). The initial conditions at $T=0$ were given by the steady solutions calculated using Wallwork et $a .^{2}$ as

$$
A(S, 0)=R_{0}^{2}(s), \quad u(S, 0)=u_{0}(s)
$$

We note that $s=\epsilon S$, so there is a dependence of the initial conditions on $\epsilon$, which results because of the full expression for the curvature being retained to ensure numerical stability. The value of $\epsilon=\frac{a}{s_{0}}$ can be measured from experiments. In the calculations we used $\epsilon=0.01$ which is typical. (For a straight uniform jet $A(S, 0)=1, u(S, 0)=1$, which corresponds to $R b=\infty$.)

We impose the upstream boundary conditions at the nozzle

$$
A(0, T)=1, \quad u(0, T)=1+\delta \sin (K T) .
$$

The amplitude of the disturbance $\delta$ can be varied to obtain the breakup length desired.

We should note also that the non-dimensional wavenumber is equivalent to disturbance frequency (see also Hilbing and Heister ${ }^{9}$ ), so $K$ (which is the disturbance frequency) fixes the 
wavelength of the perturbation and, following the linear stability analysis, we need $K<1$ to have unstable jets. We can also change the initial conditions, to make an initial perturbation of radius, rather than of velocity. The downstream boundary conditions are obtained by quadratic extrapolation of the last internal mesh points. We have carried extensive checks to verify that the choice of these boundary conditions do not affect the solution upstream, except when the Weber number is very small (when absolute instability may occur, see Lin and Reitz $\left.{ }^{32}\right)$.

In the simulations we choose that the jet-breakup occurs when the minimum dimensionless radius of the jet is less then a small value, arbitrarily chosen (usually $5 \%$ of the initial radius). Downstream of breakup point, the jet solution no longer has physical meaning, since the jet in that region will have broken up into droplets, which cannot be described by this approach, as is also the case in other works. ${ }^{6-8,11}$

The solutions obtained can be put on the physical plane $x-z$ in the following way: -for each $S \geq 0$ we have the steady centreline calculated from Wallwork et al. ${ }^{2} X(S):=$ $X(s), Z(S):=Z(s)$ where $s=\epsilon S$. We also can obtain $X^{\prime}(S), Z^{\prime}(S)$;

-for each $S \geq 0$ we calculate, by the numerical method described above, $R(S, T)$ at a certain time $T$;

-the equations of the free surface of the jet on the $x-z$ plane are then $\left(X(S) \pm Z^{\prime}(S) \times\right.$ $\left.R(S, T), Z(S) \mp X^{\prime}(S) \times R(S, T)\right)$ for each $S$.

\section{NUMERICAL RESULTS}

The range of the numerical parameters used in simulations was:

$$
n=800, \ldots, 4800, d S=0.2, \ldots, 0.025, d T=10^{-2}, \ldots, 10^{-5}
$$

The numerical accuracy was checked by varying those parameters. Our numerical method has also been compared with an implicit Crank-Nicholson method and a very good agreement was found. We also checked the method in the case of axisymmetric jets.

The effect of the wavenumber $K$ of the disturbance on the breakup of the jet is presented in Fig. 2(a). We show the last stage of the jet, before the breakup for $W e=100, R b=10$ obtained with 3200 points. The spatial grid was chosen $d S=0.05$ and the time step $d T=10^{-4}$. The initial disturbance has the magnitude $\delta=0.01$. (As previously discussed, 
the disturbance is weakly nonlinear when $\delta \approx \gamma$. Hence we have chosen a disturbance at the orifice which is smaller than this, so that the travelling wave will initially be linear before it grows and becomes nonlinear.) We can see that for small values of $K$ a thin thread is formed between two main droplets which will give birth to a satellite drop. The size of the satellite drops becomes smaller as the wavenumber is increased. We can see that the jet is most unstable for $K$ between 0.7 and 0.9. This fits with the results of Wallwork et al. ${ }^{2}$ when the most unstable wavenumber occurs for $K=0.697$, which is also the case of a straight jet. ${ }^{27}$ We should note that the growth of unstable modes is just the same as the temporal growth, for large Weber number..$^{2,33}$

It is worth noting that the figure has a horizontal exaggeration, which may distort the images of the jet shapes. (Note that the horizontal and vertical axes on fig. 2(a) are not of the same length.) We used this to present the evolution of the disturbances of the whole jets, from the orifice to the breakup point. A close-up of the breakup for $K=0.9$ is presented with the natural scaling in Fig. 2(b). However, if all figures in this paper were shown in this way for the whole length of each jet, each figure would need to be unreasonably large throughout the paper, since each jet is slender.

In Fig. 3 the breakup length of the jet is presented as a function of the wave number $K$ for two different values of $\delta$. It can be observed that there is a minimum of the breakup length corresponding to the most unstable wavenumber. The breakup length is also affected by the initial disturbance magnitude. We see that as $\delta$ increases, the most unstable wavenumber increases from 0.697 which is the limiting value for $\delta \rightarrow 0 .^{2}$

Obviously, this method neglected the equation (12) which is used only to calculate the steady (initial) solutions. For $T=0$ the equation (12) is satisfied identically, but for $T>0$ only approximately. We can input the calculated solutions at a time $T>0$ in the equation to see how the error evolves in time. In Fig. 4 the value of the function on the left-hand side of $(12)$ is presented for two times, one close to breakup (which occurs at $s \approx 1$ ) and one about half-way towards breakup against the arclength $s$. The equation, which is a solvability condition at a higher order in the perturbation analysis, was used to calculate the initial steady conditions for our nonlinear evolution problem. This graph can be viewed as the error which resulted from the neglect of this equation as the time evolves. If the translational velocity terms $v_{0}$ and $w_{0}$ had been retained in the equations of motion, as well as allowing $X_{0 t} \neq 0$ and $Z_{0 t} \neq 0$, then these terms would appear on the right-hand side of (12). Hence 
this error is a measure of the size of the translational velocity components $v_{0}$ and $w_{0}$, and hence the size of this temporal translational velocity of the jet's centreline.

The error $E$ of neglecting these translational velocity components can be considered as $E=Z_{s} X_{t}-Z_{t} X_{s}$. In section $\mathrm{V}$ an equation (26) is derived for $E$. It can be seen that $E$ satisfies a first order inhomogenous partial differential equation and the inhomogenous part of that equation is given by the left hand-side of (12). Therefore Figure 4 shows the magnitude of the forcing imposed on the error $E$. Since $E$ is proportional to the translational velocity components $v_{0}$ and $w_{0}, E$ should be measured in relation to the mean jet speed $u_{0}$, which is initially close to one and grows as $S$ and $T$ grow.

The evolution of a disturbance for various time steps is shown in Fig. 5. We can see that for small value of $T$, the disturbance is almost invisible, but it grows when $T$ approaches the breakup time. Also the disturbance can be seen travelling down the jet.

In Fig. 6 we present two different solutions for two different Rossby numbers $R b$, the others parameters being identical. The breakup length is slowly increasing with the Rossby number for $R b$ greater than 0.93, when the Weber number is constant (see Fig. 7). At $R b \approx 0.93$ the breakup occurs simultaneously at two points, and for $R b$ smaller than this critical value we see again that the breakup length increases with the Rossby number. This behaviour was not predicted by the linear theory. We can therefore state that there is no monotonic relationship between breakup length and $R b$.

In Fig. 8 the influence of the surface tension is presented. The Rossby number is kept constant $(R b=10)$ and the Weber number is varied. As Weber number is decreased the breakup lengths are shorter, as in the straight axisymmetric case. The breakup length against the Weber number is plotted in Fig. 9.

The numerical simulations can be compared with experiments already carried out. The experimental setup is described in Ref. 25. We present in Fig. 10 a comparison between a simulation and a photograph of a rotating water jet at identical parameter values. Qualitative agreement can be seen between these results in the shape of the jet close to breakup. In our numerical simulation we could select $\delta$ to match the breakup lengths exactly, using $\delta$ as a fitting parameter with very similar results. (It was shown in Ref. 23 that for linear jets it is always possible to choose $\delta$ to match experimental and theoretical results for breakup lengths in any given case, and so this will also be the case for nonlinear jets. However, there seems little point in doing so since $\delta$ is an unknown parameter unless experimental data is 
available for fitting.) Here we have not chosen to select $\delta$ by fitting the experimental and theoretical breakup lengths, but instead show that for a reasonable small value of $\delta$ that gives a breakup length of the correct order of magnitude in the numerical simulation, the shape of the jet at breakup appears similar between theory and experiments, even though the breakup lengths to not match exactly. Therefore, the qualitative shape of the jet at breakup is robust to changes in $\delta$, and is not highly dependent upon determining the value of $\delta$ from experimental fitting. Therefore, this model will be useful for estimating breakup shape, drop sizes etc, even when $\delta$ is not known exactly. (The physical scale of $1 \mathrm{~cm}$ is shown on Fig. 10(a). A length of $1 \mathrm{~cm}$ on Fig. 10(b) corresponds to a non-dimensional axis length of 0.2 units, for comparison.)

Figure 11 shows $R_{0}$ plotted against $s$ for two different values of $R b$, with all other parameters held constant. This demonstrates the role of the initial conditions, which depend upon the rotation rate, on the solution. It can be seen therefore that the rotation rate of the container $\Omega$ does not only alter the trajectory of the jet but also the shape of the waves and the breakup length. After the breakup point on both jets, which is at $s=0.19$ for $R b=1$, and $s=0.34$ for $R b=10$, the initial condition can be seen clearly on these graphs. (This part of the jet has been undisturbed by the travelling waves which have not been able to propagate sufficiently upstream without breaking before reaching the latter end of the jets. Of course this part of the jet is unphysical since the breakup point of the jet is obviously the physical end of the jet, and it was included only from numerical reasons.)

In all the cases considered it was observed that the breakup occurs asymmetrically, similar to the case of a straight jet with the formation of a cone-like structure close to the breakup point. A similar phenomenon was noted in Ref. 34. We believe that the computational breakup will be similar to the breakup of a straight inviscid jet (see Papageorgiou and Orellana ${ }^{7}$ ). Equation (12) shows that since $R_{0} \rightarrow 0$ and $u_{0} \rightarrow \infty$ at breakup then the jet will be locally straight at the breakup point and $X$ and $Z$ should not become singular.

\section{LONG WAVES AND UNSTEADY TRAJECTORY}

In this section we will consider very long waves with $\lambda \sim s_{0}$ (so that $\gamma \sim \epsilon$ ) and allow the centreline to be time-dependent $\left(X_{t} \neq 0, Z_{t} \neq 0\right)$. Therefore the expansions in $v$ and $w$ must contain leading-order terms. To simplify the analysis we will consider all the variables 
depending only in long lengthscales and all the expansions will be in $\epsilon$ (and not in $\gamma$ ). Using the slender jet assumptions we put

$$
\begin{gathered}
u=u_{0}(s, t)+O\left(\epsilon^{2}\right), v=v_{0}(s, \phi, t)+\epsilon n v_{1}+\ldots, w=w_{0}(s, \phi, t)+\epsilon n w_{1}+\ldots \\
R=R_{0}(s, t)+\epsilon R_{1}+\ldots, X=X_{0}(s, t)+\epsilon X_{1}+\ldots, Z=Z_{0}(s, t)+\epsilon Z_{1}+\ldots, p=p_{0}(s, t)+\epsilon p_{1}(s, \phi, n, t)+\ldots
\end{gathered}
$$

We write again $X_{0}$ and $Z_{0}$ as $X$ and $Z$ respectively. The full equations which include the azimuthal velocity $w$ can be found in Wallwork et al. ${ }^{2}$

From the kinematic condition at leading order we obtain

$$
v_{0}(s, \phi, t)=\left(Z_{s} X_{t}-Z_{t} X_{s}\right) \cos \phi
$$

and from the conservation of mass at leading order, after integration, we obtain

$$
w_{0}(s, \phi, t)=-\left(Z_{s} X_{t}-Z_{t} X_{s}\right) \sin \phi
$$

We denote $E=Z_{s} X_{t}-Z_{t} X_{s}$. The first Euler equation at order $\epsilon$ can be written as

$$
\begin{gathered}
u_{0 t}+E\left(Z_{s t} X_{s}-X_{s t} Z_{s}\right)+u_{0} u_{0 s}+u_{0}\left(X_{s} Z_{s s}-X_{s s} Z_{s}\right) E= \\
=-p_{0 s}+\frac{2 E}{R b}+\frac{(X+1) X_{s}+Z Z_{s}}{R b^{2}} .
\end{gathered}
$$

After some computations, and using the second and the third Euler equation, we obtain from the kinematic condition at order $\epsilon$

$$
R_{0 t}+\frac{u_{0 s}}{2} R_{0}+\frac{\left(X_{s} Z_{s s}-X_{s s} Z_{s}\right)}{2} E R_{0}+u_{0} R_{0 s}-R_{0 s}\left(X_{t} X_{s}+Z_{t} Z_{s}\right)=0 .
$$

and

$$
v_{1}(s, \phi, t)=-\frac{u_{0 s}}{2}-\frac{\left(X_{s} Z_{s s}-X_{s s} Z_{s}\right)}{2} E, \quad w_{1}(s, \phi, t)=w_{1}(s, t) .
$$

The expression of $p_{1}$ is found to be

$$
\begin{gathered}
p_{1}(s, \phi, n, t)=-n \cos \phi\left[E_{t}+u_{0}\left(X_{s t} Z_{s}-Z_{s t} X_{s}\right)-\frac{E}{2}\left(u_{0 s}+\right.\right. \\
\left.+\left(X_{s} Z_{s s}-X_{s s} Z_{s}\right) E\right)+u_{0} E_{s}-\left(X_{s} Z_{s s}-X_{s s} Z_{s}\right) u_{0}^{2}+\frac{2 u_{0}}{R b}- \\
\left.-\frac{1}{R b^{2}}\left((X+1) Z_{s}-Z X_{s}\right)\right]-n \sin \phi E w_{1}+p_{11}(s, t) .
\end{gathered}
$$

The dynamic condition can be rewritten

$$
p_{\mid n=R}=\frac{1}{R_{0} W e}+\epsilon\left(-\frac{R_{1 \phi \phi}+R_{1}}{R_{0}^{2}}+\cos \phi\left(X_{s} Z_{s s}-X_{s s} Z_{s}\right)\right) W e^{-1}+O\left(\epsilon^{2}\right),
$$


and we obtain

$$
p_{0}(s, t)=\frac{1}{R_{0} W e}
$$

and, after using solvability condition as in Section II,

$$
\begin{gathered}
E_{t}+u_{0}\left(X_{s t} Z_{s}-Z_{s t} X_{s}\right)+E\left(-\frac{u_{0 s}}{2}-\left(X_{s} Z_{s s}-X_{s s} Z_{s}\right) \frac{E}{2}\right)+u_{0} E_{s}= \\
\left(X_{s} Z_{s s}-X_{s s} Z_{s}\right) u_{0}^{2}-\frac{2 u_{0}}{R b}+\frac{1}{R b^{2}}\left((X+1) Z_{s}-Z X_{s}\right)-\frac{X_{s} Z_{s s}-X_{s s} Z_{s}}{R_{0} W e} .
\end{gathered}
$$

The right-hand side of this equation is exactly the equation (12). To find $X(s, t)$ and $Z(s, t)$ this equation can be solved along with (15). But since the right hand side of (26) is always very small (except at the time and location of breakup when it is only moderately small) there will usually be little utility in actually doing so since $E \approx 0$ is a good approximation to the solution. Experiments suggest that this is so for low viscosity liquids but not for highly viscous liquids: this will be the subject of a future paper.

If $X_{0}$ and $Z_{0}$ are not time-dependent and $s$ and $t$ are replaced by $\bar{s}$ and $\bar{t}$ in derivatives of $u_{0}$ and $R_{0}$, then equations (15), (24), (25) and (26) reduce to the model from section II at leading-order, namely (12 - 15), as should be expected. Therefore the model already presented in previous sections is a good model unless the waves are unusually long or the trajectory is thought to be time-dependent.

We solve the equations from this section by extending the numerical method from section III. We solve (15), (24), (25) and (26) using the same initial and boundary conditions as before. Generally the results are identical to those already presented for the above mentioned reasons, so we concentrate on just a couple of examples. Figure 12 shows the perturbation of the trajectory of the jet from its initial condition plotted against $s$ for various times $t$ for a short jet disturbance with $K=0.697$. The solid curves show the perturbation of $X_{0}$ from its initial state and the dashed lines show $Z_{0}$ from its initial state. Note the maximum deviation is of the order of $10^{-3}$ and hence small compared to $X_{0}(s)$ and $Z_{0}(s)$. This is typical of all results attempted here. Hence the trajectory is effectively steady in the frame of reference moving with the container for an inviscid jet, which agrees with experimental observations. Also note that the motion for $X_{0}$ and $Z_{0}$ is not wave-like on a short lengthscale. The breakup point is close to $s=1$ in this case.

Figure 13 shows two different jets. One is nearly straight with a short disturbance. This jet is the same as if described by either the model in this section or the one from section II. 
(The numerical results are indistinguishable.) The other jet is highly curved and shows a very long wave disturbance propagating upstream, where the wavenumber $K$ is small. This jet disturbance is different to what would be produced if the model from section II were used instead, since the last extra term on the right-hand side of (24) is not small in this case even if the trajectory is steady. Hence this long wave receives an extra acceleration which would not be present in the previous model. However, this jet would be difficult to see in experiments since this is not the fastest growing mode. It should be noted that the trajectory is still almost steady even in this case, as in Figure 12.

\section{CONCLUSIONS}

The evolution of a disturbance on a rotating, slender inviscid jet emanating from a small orifice up to the breakup point has been studied. The nonlinear partial differential equations which describe this evolution were obtained by using a multiple scale asymptotic approach. The numerical method used to solve the system was described and the influence of various parameters on the solutions have been quantified.

Two different models have been presented. The first is the simpler of the two, with rotation only entering the formulation via the initial conditions. It has been shown that rotation still plays a vital role in the dynamics of the jet, affecting trajectory, breakup length, droplet size and wave shape. The results clearly agree qualitatively with experimental observations. The second model is more complicated, allowing an unsteady trajectory. The nature of the dynamics of the trajectory have been described, and are not found to be wave-like. This model is only important for a viscous jet if very long waves are forced.

The one-dimensional approach is justified in the inviscid case as the surface tension will suppress some short-scale motions in the jet cross section, as observed in the experimental photographs of Wong et $a l^{26}$ and Partridge $e t a l^{35}$, where the jet appears axi-symmetric even for water which has a small viscosity. Also, using Wallwork et al. ${ }^{2}$, the results of linear instability calculations with multiple scales on inviscid jets, without assuming the jet is quasi-one-dimensional, agree with the linear instability results of the quasi-one-dimensional jet numerically to about 1 to 2 significant figures for values of the most unstable wavenumber (also see Section III here). This is also the case for straight inviscid jets, where the most unstable wavenumber from the quasi-one-dimensional model is 0.707 in non-dimensional 
units (correct to 3 significant figures), which compares fairly well to the equivalent result of 0.697 obtained without making the quasi-one-dimensional assumption for a straight inviscid liquid jet. See Partridge ${ }^{36}$ for further details. If the inviscid model is reasonable for a linear instability calculation, then it should also be for a nonlinear calculation.

The methods developed here can in principle be applied for other physical problems. For example the full three-dimensional problem with both gravity and rotation can be studied in the same manner. Also the viscous case could be treated using a similar method. These will be considered in future papers.

\section{Acknowledgments}

E. Părău and D.C. Wong thank EPSRC for financial support and Lucy Partridge for some help in preparing this document.

\section{Appendix}

We compare in this Appendix the quasi-one-dimensional equations derived by Entov \& Yarin $^{18}$ for a bending jet with the equations obtained in our asymptotic derivation. Their quasi-one dimensional equations, when the jet axis is a curve lying in a plane [Entov \& Yarin $^{18}$ p. 101. eq (4.17)], are

$$
\begin{gathered}
\frac{\partial \lambda f}{\partial t}+\frac{\partial f W}{\partial \breve{s}}=0, \\
\frac{\partial \lambda f V_{\tau}}{\partial t}-\frac{f V_{n}}{\lambda} \frac{\partial \lambda V_{n}}{\partial \breve{s}}+\frac{\partial f V_{\tau} W}{\partial \breve{s}}-\lambda f W k V_{n}=\frac{1}{\rho} \frac{\partial P}{\partial \breve{s}}+\lambda f F_{\tau}+\frac{1}{\rho} \lambda q_{\tau}, \\
\frac{\partial \lambda f V_{n}}{\partial t}+\frac{f V_{\tau}}{\lambda} \frac{\partial \lambda V_{n}}{\partial \breve{s}}+\frac{\partial f V_{n} W}{\partial \breve{s}}+\lambda f W k V_{\tau}=\frac{1}{\rho} P+\lambda f F_{n}+\frac{1}{\rho} \lambda q_{n}, \\
\lambda=\left[1+\left(\frac{\partial H}{\partial \breve{s}}\right)^{2}\right]^{1 / 2}, \quad k=\frac{\partial^{2} H}{\partial s^{2}} \lambda^{-3}, \quad \frac{\partial H}{\partial t}=\lambda V_{n}, \quad W=V_{\tau}-V_{n} \frac{\partial H}{\partial \breve{s}} .
\end{gathered}
$$

Here $f=\pi R^{2}(\breve{s}, t)$ is the area of the jet cross-section and $F_{\tau}, F_{n}$ are the components of the external force $F$ per unit mass. The resultant action of the external loads is specified by the linear density of forces $q$ applied to the jet axis and is taken to be zero.

Assuming the jet motion to be such that the tangent to the jet axis at any instant and at all points makes an acute angle with a straight line $O_{1} \xi$, the jet axis in their case is described 
in the Cartesian coordinate by

$$
\mathbf{R}=\mathbf{i} \breve{s}+\mathbf{k} H(\breve{s}, t)
$$

while our jet axis, which does not have to satisfy such a restriction, is

$$
\mathbf{R}=\mathbf{i} X(s, t)+\mathbf{k} Z(s, t) .
$$

The relations between the coordinates systems is

$$
\breve{s}=X(s, t), H(\breve{s}, t)=Z(s, t),
$$

and it follows $\frac{\partial H}{\partial \breve{s}}=\frac{Z_{s}}{X_{s}}, \lambda=\frac{1}{X_{s}}, k=Z_{s s} X_{s}-X_{s s} Z_{s}, \frac{\partial H}{\partial t}=Z_{t}-\frac{Z_{s}}{X_{s}} X_{t}=-\frac{E}{X_{s}}$. Generally, for a function $\breve{a}(\breve{s}, t)=\breve{a}(X(s, t), t):=a(s, t)$, we have $\frac{\partial \breve{a}}{\partial \breve{s}}=\frac{1}{X_{s}} \frac{\partial a}{\partial s}, \frac{\partial \breve{a}}{\partial t}=\frac{\partial a}{\partial t}-\frac{X_{t}}{X_{s}} \frac{\partial a}{\partial s}$.

The velocities $V_{\tau}$ and $V_{n}$ are the components of the velocity fields on the centreline, in tangential and normal direction

$$
\tau=\mathbf{e}_{\mathbf{s}}=X_{s} \mathbf{i}+Z_{s} \mathbf{k} \quad \text { and } n=-Z_{s} \mathbf{i}+X_{s} \mathbf{k},
$$

and are found to be $V_{\tau}=u, V_{n}=-E$.

The longitudinal force $P$ on the cross-section becomes in the inviscid case (see Entov\& Yarin $^{18}$, eq. 4.16 , after neglecting the viscous part and a few manipulations)

$$
P=\sigma \pi R^{2}\left(\frac{1}{R\left(1+R_{s}^{2}\right)^{1 / 2}}+\frac{R_{s s}}{\left(1+R_{s}^{2}\right)^{3 / 2}}\right) .
$$

The equations (27-(29) can be rewritten as

$$
\begin{gathered}
\frac{\partial}{\partial t}\left(\frac{R^{2}}{X_{s}}\right)-\frac{X_{t}}{X_{s}} \frac{\partial}{\partial t}\left(\frac{R^{2}}{X_{s}}\right)+\frac{1}{X_{s}} \frac{\partial}{\partial s}\left(R^{2}\left(u+E \frac{Z_{s}}{X_{s}}\right)\right)=0 \\
\frac{\partial}{\partial t}\left(\frac{R^{2} u}{X_{s}}\right)-\frac{X_{t}}{X_{s}} \frac{\partial}{\partial s}\left(\frac{R^{2} u}{X_{s}}\right)-R^{2} E \frac{\partial}{\partial s}\left(\frac{E}{X_{s}}\right)+\frac{1}{X_{s}} \frac{\partial}{\partial s}\left(R^{2} u\left(u+E \frac{Z_{s}}{X_{s}}\right)\right) \\
\quad+\frac{1}{X_{s}} R^{2}\left(u+E \frac{Z_{s}}{X_{s}}\right)\left(X_{s} Z_{s s}-X_{s s} Z_{s}\right) E=\frac{1}{\rho} \frac{\partial}{\partial s}\left(\frac{P}{\pi X_{s}}\right)+\frac{R^{2}}{X_{s}} F_{\tau} \\
\frac{\partial}{\partial t}\left(\frac{R^{2} E}{X_{s}}\right)-\frac{X_{t}}{X_{s}} \frac{\partial}{\partial s}\left(\frac{R^{2} E}{X_{s}}\right)+R^{2} u \frac{\partial}{\partial s}\left(\frac{E}{X_{s}}\right)+\frac{1}{X_{s}} \frac{\partial}{\partial s}\left(R^{2} E\left(u+E \frac{Z_{s}}{X_{s}}\right)\right) \\
-\frac{1}{X_{s}} R^{2}\left(X_{s} Z_{s s}-X_{s s} Z_{s}\right) u\left(u+E \frac{Z_{s}}{X_{s}}\right)=\frac{1}{\rho}\left(X_{s} Z_{s s}-X_{s s} Z_{s}\right) \frac{P}{\pi}+\frac{R^{2}}{X_{s}} F_{n} .
\end{gathered}
$$

We introduce the short-scale dimensionless variables $\bar{s}, \bar{t}$ and use the scaling from the section II. After using our asymptotic expansion, at leading order $R$ can be replaced by 
$R_{0}$ and $u$ by $u_{0}$. Assuming that the centreline is not time-dependent at leading order, it can be observed that the equation (30) is exactly the equation (13) multiplied by $2 R_{0} / X_{s}$. Again, by multiplying the equation (14) with $R_{0}^{2} / X_{s}$ and subtracting the equation (30), we will obtain equation (31), with the mention that $F_{\tau}$ will appear at higher order in our analysis and we obtain on the right hand side only the leading order term $-\frac{1}{W e R_{0}}$ instead of the pressure term $P_{s}$ containing the full curvature, which is a common problem for any asymptotic analysis (see Eggers ${ }^{11}$ ). The solvability condition (12) can be recovered from the equation (32), with the same observation as above, with the term $-\frac{\left(X_{s} Z_{s s}-X_{s s} Z_{s}\right)}{W e R_{0}}$ instead of $\frac{\left(X_{s} Z_{s s}-X_{s s} Z_{s}\right)}{W e} P$ corresponding to full curvature.

A comparison between the above equations and our equations obtained when the centreline is time-dependent for long-wave disturbances (section V) can also be performed, with similar conclusions. 
* Present address: School of Mathematics, University of East Anglia, Norwich, NR4 7TJ, UK

$\dagger$ Author to whom the correspondence should be addressed. Electronic mail: decentsp@for.mat.bham.ac.uk. Telephone + 44 (0) 121414 7566. Fax + 44 (0) 1214143389.

1 K.G. Andersen and G. Yttri, "Et fors $\phi k$ verdt," Univesitetsforlaget Oslo (1997).

2 I.M. Wallwork, S.P. Decent, A.C. King and R.M.S.M. Schulkes, "The trajectory and stability of a spiralling liquid jet. Part 1. Inviscid theory," J. Fluid Mech. 459, 43 (2002).

3 S. Decent, A.C. King, I.M. Wallwork, "Free jets spun from a prilling tower," J. Eng. Math. 42, 265 (2002).

4 H. C. Lee, "Drop formation in a liquid jet," IBM J. Res. Dev. 18, 364 (1974).

5 N. N. Mansour, T. S. Lundgren, "Satellite formation in capillary jet break-up," Phys. Fluids A 2, $1141(1990)$.

6 R.M.S.M. Schulkes, "Dynamics of liquid jets revised," J. Fluid Mech. 250, 635 (1993).

7 D.T. Papageorgiou and O. Orellana, "Study of cylindrical jet breakup using one-dimensional models of the Euler equations," SIAM J. Appl. Math. 59, 286 (1998).

8 J. Eggers and T. F. Dupont, "Drop formation in a one-dimensional approximation of the NavierStokes equation," J. Fluid Mech. 246, 205 (1994).

9 J.H. Hilbing, S.D. Heister, "Droplet size control in liquid jet breakup," Phys. Fluids 8, 1574 (1996).

10 L. Cheong, "Droplet Formation from Capillary Jet Instability Under Low Flows," Ph.D. thesis, University of Queensland (2000).

11 J. Eggers, "Nonlinear dynamics and breakup of free-surface flows," Rev. Mod. Phy. 69, 865 (1997).

12 J.-M. Vanden-Broeck, "Water Waves and Related Free-Surface Flows," Proceeding of IUTAM Symposium on Free-Surface Flows, Birmingham, July 2000 (Kluwer Academic Publishers, Dordrecht, 2001).

13 C. Weber, "Zum Zerfall eines Flussigkeitesstrables", Z. Angew. Math. Mech. 11, 136 (1931).

14 J.-M. Vanden-Broeck and J.B. Keller, "Jet rising and falling under gravity," J. Fluid Mech. 124, 335 (1982).

15 F. Dias and J.M. Vanden-Broeck, "Flows emerging from a nozzle and falling under gravity," 
J. Fluid Mech. 213, 465 (1990).

16 D.S. Finnicum, S.J. Weinstein and K.J. Ruschak, "The effect of applied pressure on the shape of a two-dimensional liquid curtain falling under the influence of gravity," J. Fluid Mech. 255, 645 (1993).

17 L.J. Cummings and P.D. Howell, "On the evolution of non-axisymmetric viscous fibres with surface tension, inertia and gravity," J. Fluid Mech. 389, 361 (1999).

18 V.M. Entov and A.L. Yarin, " The dynamics of thin liquid jets in air," J. Fluid Mech. 140, 91 (1984).

19 A.L. Yarin, Free liquid jets and films: Hydrodynamics and Rheology (Longman, 1993).

20 D.H. Reneker, A.L. Yarin, H. Fong, S. Koombhongse, "Bending instability of electrically charged liquid jets of polymer solutions in electrospinning," J. Appl. Phys.87, 4531 (2000).

21 A.L. Yarin, S. Koombhongse, D.H. Reneker, "Taylor cone and jetting from liquid droplets in electrospinning of nanofibers," J. Appl. Phys. 90, 4836 (2001).

22 M.M. Hohman, M. Shin, G. Rutledge, M.P. Brenner, "Electrospinning and electrically forced jets. I. Stability theory," Phys. Fluids. 13, 2201 (2001).

23 I.M. Wallwork, "The trajectory and stability of a curved liquid jet," Ph. D. Thesis, Univ. of Birmingham (2002).

24 A.D.D. Craik, Nonlinear wave interactions in fluid flows. (Cambridge, 1989)

25 D.C.Y. Wong, M.J.H. Simmons, S.P. Decent, A.C. King, E.I. Părău, "Dynamic break-up and drop formation from a liquid jet spun from a rotating orifice. Part I: Experimental," Proc. of FEDSM'03: 4th ASME-JSME Joint Fluids Engineering Conference, Honolulu, Hawaii, USA, July, 2003.

26 D.C.Y. Wong, M.J.H. Simmons, S.P. Decent, E.I. Părău, A.C. King, "Break-up dynamics and drop size distributions created from spiralling liquid jets," Int. J. of Multiphase Flow 30, 499 (2004).

27 Lord Rayleigh, On the instability of jets, Proc. London Math. Soc., 10, 4 (1878).

28 A. F. Nayfeh, Introduction to perturbation techniques (Wiley, 1993).

29 Y. Zhu, H.N. Oğuz and A. Prosperetti, "On the mechanism of air entrainemnt by liquid jets at a free surface," J. Fluid Mech. 404, 151 (2000).

30 Y. Li and P.D. Sclavounos, "Three-dimensional nonlinear solitary waves in shallow water generated by an advancing disturbance," J. Fluid Mech. 470, 383 (2002). 
31 W. H. Press, S.A. Teukolsky, W.T. Vetterling, B.P. Flannery, Numerical Recipes in Fortran r7: The Art of Scientific Computing; (Fortran Numerical Recipes, Vol 1) (Cambridge University Press, 2001).

32 S.P. Lin and R.D. Reitz, "Drop and spray formation from a liquid jet," Annu. Rev. Fluid Mech. 30, 85 (1998).

33 J.B. Keller, S.I. Rubinow and Y.O. Tu, "Spatial instability of a jet," Phys. Fluids. 16, 2052 (1973).

34 D.H. Peregrine, G. Shoker and A. Symon, "The bifurcation of liquid bridges," J. Fluid Mech. 212, 25 (1990).

35 L. Partridge, D.C.Y. Wong, M.J.H. Simmons, E.I. Părău, S.P. Decent "Experimental and theoretical description of the break-up of curved liquid jets in the prilling process," Chem. Eng. Res. Des. 83(A11), 1267 (1990).

36 L. Partridge," Experimental and theoretical analysis of prilling," Ph. D. Thesis, Univ. of Birmingham (2006). 
FIG. 1. Sketch of a typical disturbance.

FIG. 2(a). The jet at the breakup time for various values of $K$. The breakup point is marked by a straight line across the middle of the jet. The nozzle is at the bottom of the figure and the cylinder rotates anti-clockwise. The horizontal exaggeration is by a factor of 4 so that the whole of each jet can be easily shown in one figure. (b) A close-up of the breakup for $K=0.9$ with natural scaling, without horizontal exaggeration.

FIG. 3. The breakup length as a function of wavenumber $K$ for $\delta=0.001$ and $\delta=0.01$. In both cases there is a minimum which corresponds to the most unstable $K$. The parameters are $W e=100$ and $R b=10$.

FIG. 4. The error calculated at each $s$ by introducing the values of the variables $R$ and $u$ calculated for the unsteady jet into the equation (12). The error is shown at two different times: $\mathrm{T}=50$ (dashed line) and $\mathrm{T}=100.0$ (full line). The latter time is close to the breakup time. The parameters are $W e=100, R b=10, K=0.5, \delta=0.01$.

FIG. 5. The evolution of the disturbance before the breakup for a fixed value of $K(=0.7$ for this example). Various times are shown.

FIG. 6. Solutions for $R b=10$ and $R b=1$. The other parameters are $W e=100, K=0.7$, $\delta=0.01$.

FIG. 7. The breakup length against the Rossby number $R b$. The other parameters are constants: $W e=100, K=0.7, \delta=0.01$. We can see that for $R b=0.93$ there is a change in the breakup.

FIG. 8. Solutions for $W e=100, W e=20, W e=10$ and $W e=5$. The other parameters are $R b=10, K=0.7, \delta=0.01$.

FIG. 9. The breakup length of the jet against the Weber number $W e$. The other parameters are the same as in Fig.8.

FIG. 10. Comparison between a rotating water jet (dynamic viscosity $\mu=0.001 \mathrm{~Pa} s$ ) in (a) and our simulation (b). The nozzle is at the bottom of the figure. The cylinder rotates 
anti-clockwise at $100 \mathrm{rpm}$. The diameter of the nozzle is $0.001 \mathrm{~m}$. The numerical parameters are $W e=82, R b=1.33, \delta=0.006, K=0.7$.

FIG. 11. $R_{0}$ as a function of $s$ for $\delta=0.0035, W e=20, k=0.697$. The solid line shows $R b=1$ and the dashed line shows $R b=10$. The breakup occur at $s=0.19$ for $R b=1$, and $s=0.34$ for $R b=10$.

FIG. 12. The deviation of $X_{0}$ and $Z_{0}$ from their initial condition plotted against $s$ for various $t$ for $\delta=0.0035, W e=20, k=0.697$. The solid lines show the deviation of $X_{0}$ and the dashed lines show deviation of $Z_{0}$. The lines move away from the central axis for larger times. The times show times half-way to breakup (1), three quarters towards breakup (2) and finally the deviations at the breakup time (3).

FIG. 13. Solutions of the model in section $\mathrm{V}$ for two different jets for $\delta=0.0035$ and $W e=20$. The jet with $R b=100$ has $K=0.697$ and is almost identical for both models. The jet with $R b=1$ has $K=0.1$. Since this jet has very long waves, the multiple scales model is inappropriate to describe these disturbances and the model in section $\mathrm{V}$ must be used. 


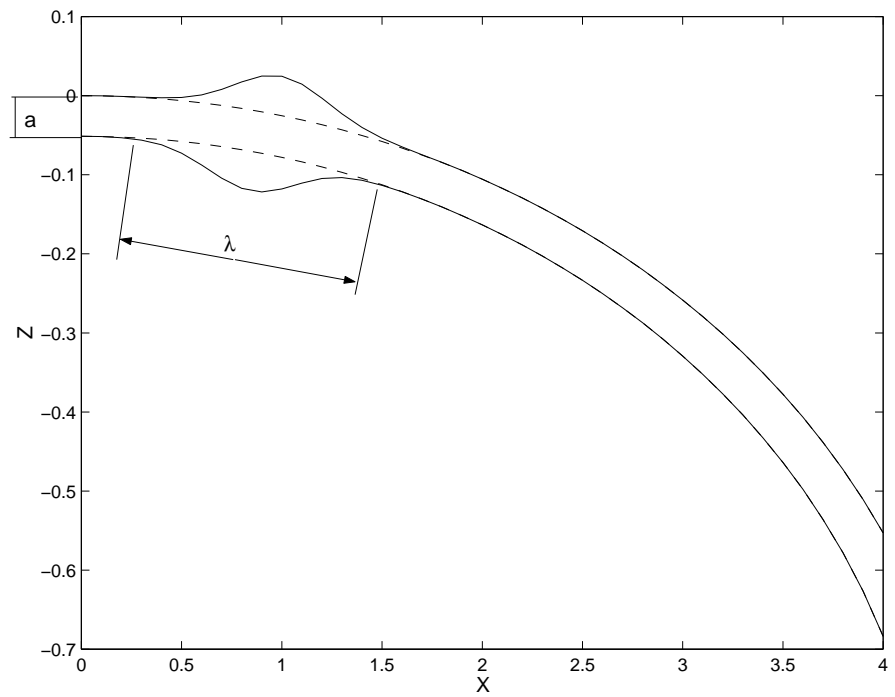

FIG. 1: Parau, Decent, King, Simmons, Wong 

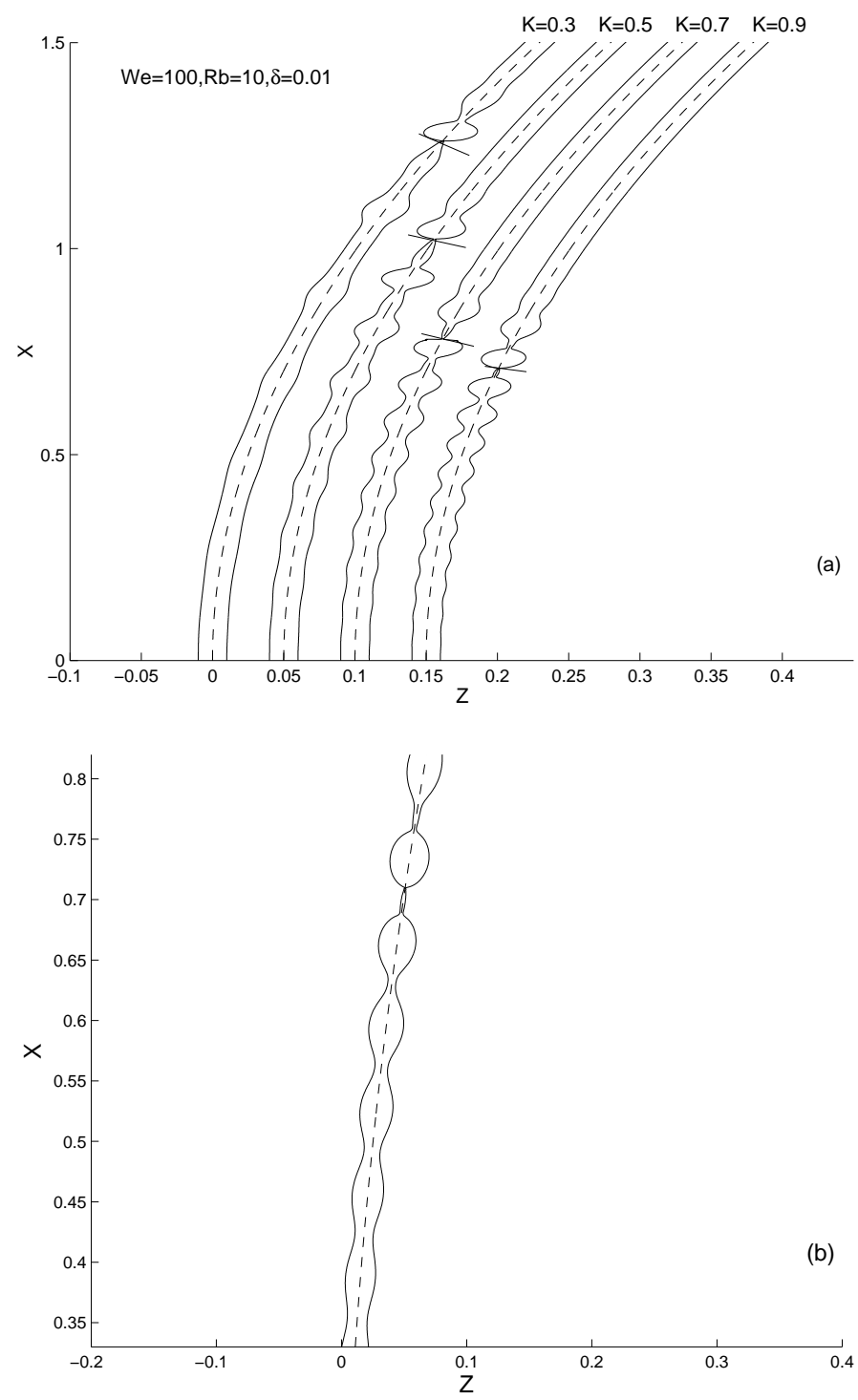

FIG. 2: Parau, Decent, King, Simmons, Wong 


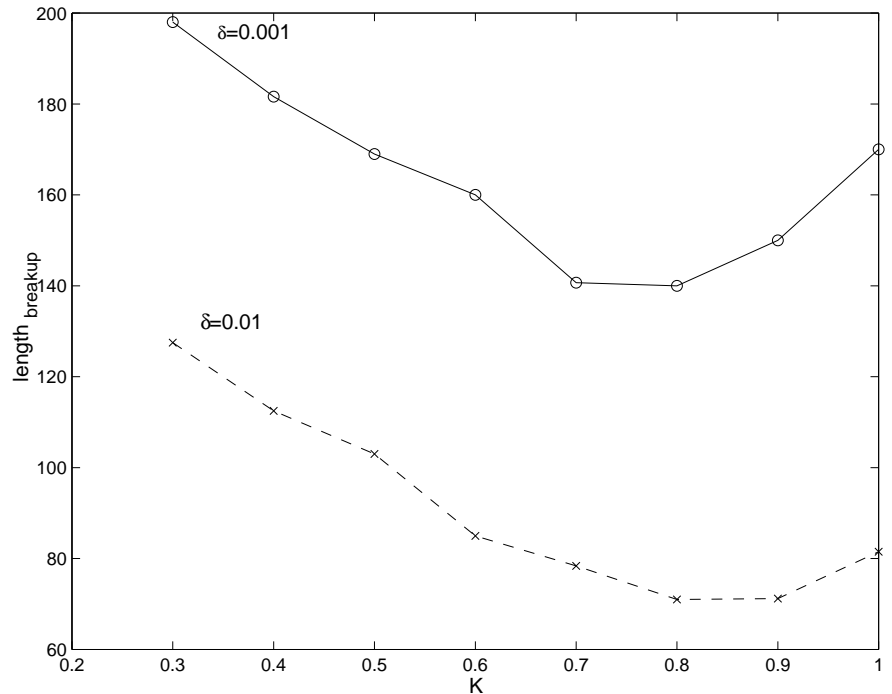

FIG. 3: Parau, Decent, King, Simmons, Wong 


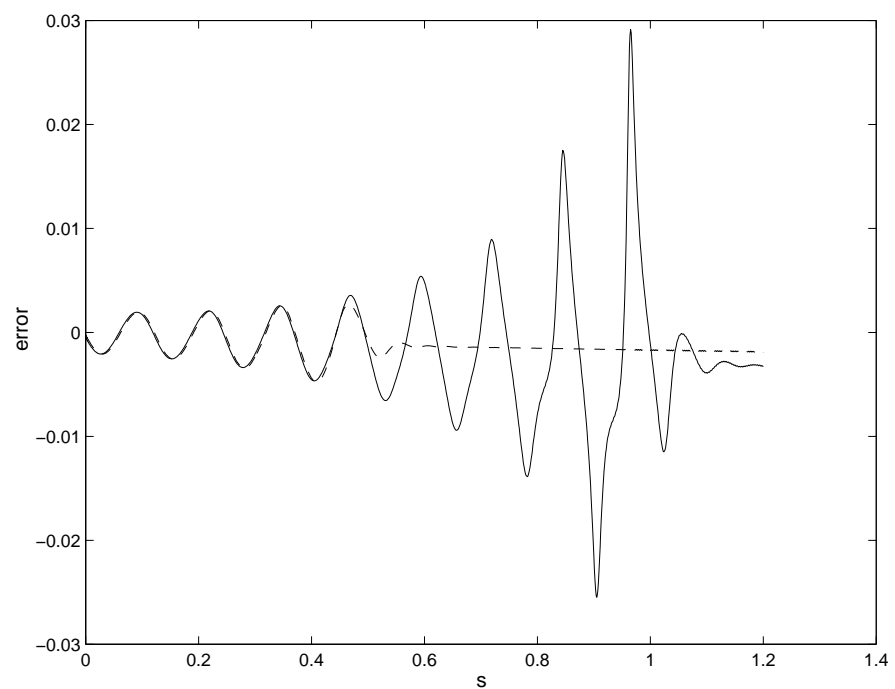

FIG. 4: Parau, Decent, King, Simmons, Wong 


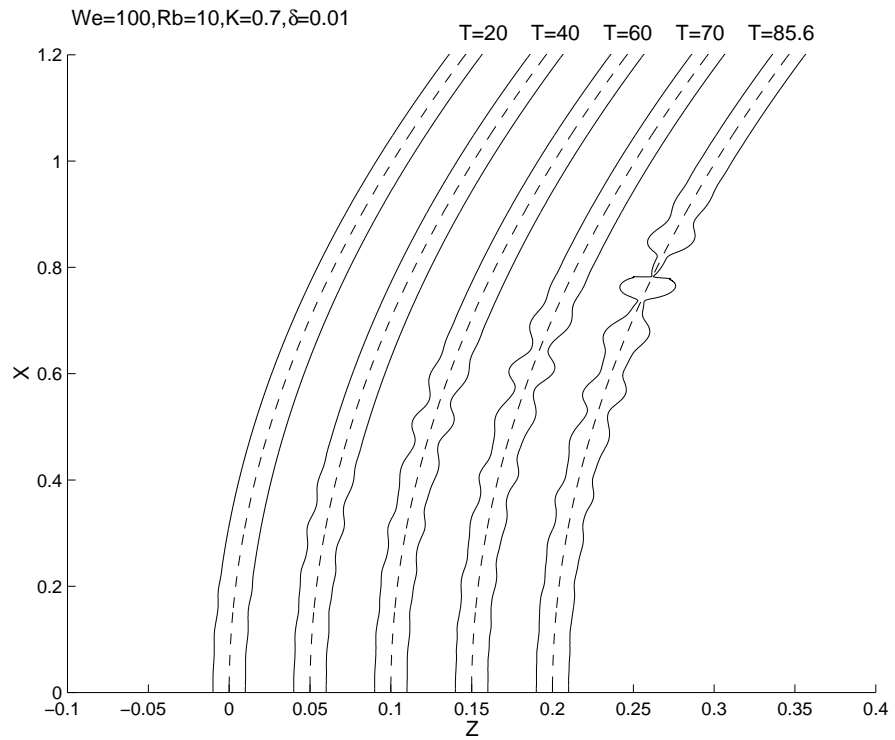

FIG. 5: Parau, Decent, King, Simmons, Wong 


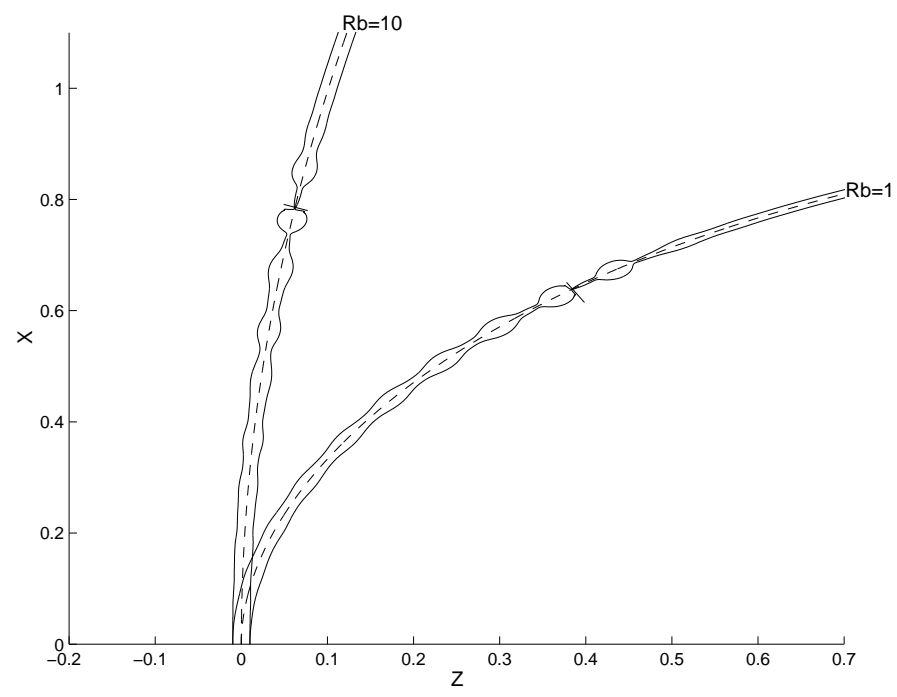

FIG. 6: Parau, Decent, King, Simmons, Wong 


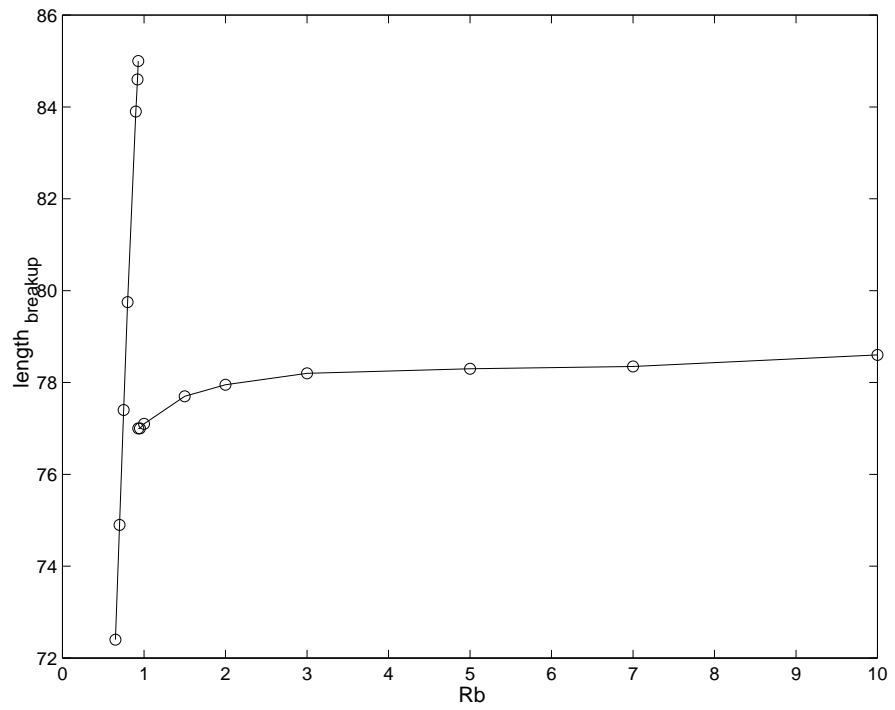

FIG. 7: Parau, Decent, King, Simmons, Wong 


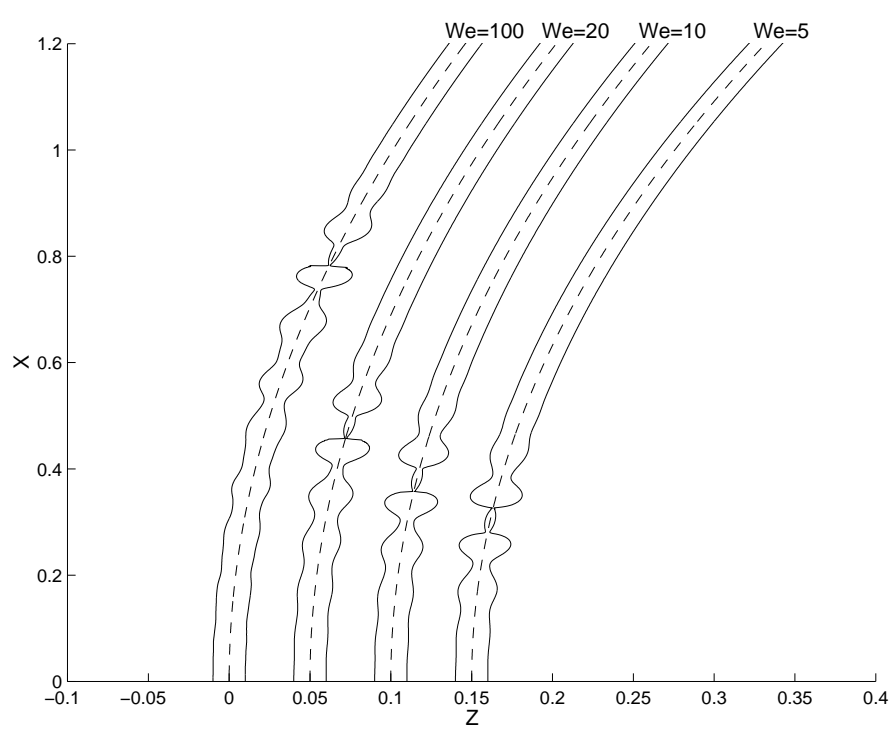

FIG. 8: Parau, Decent, King, Simmons, Wong 


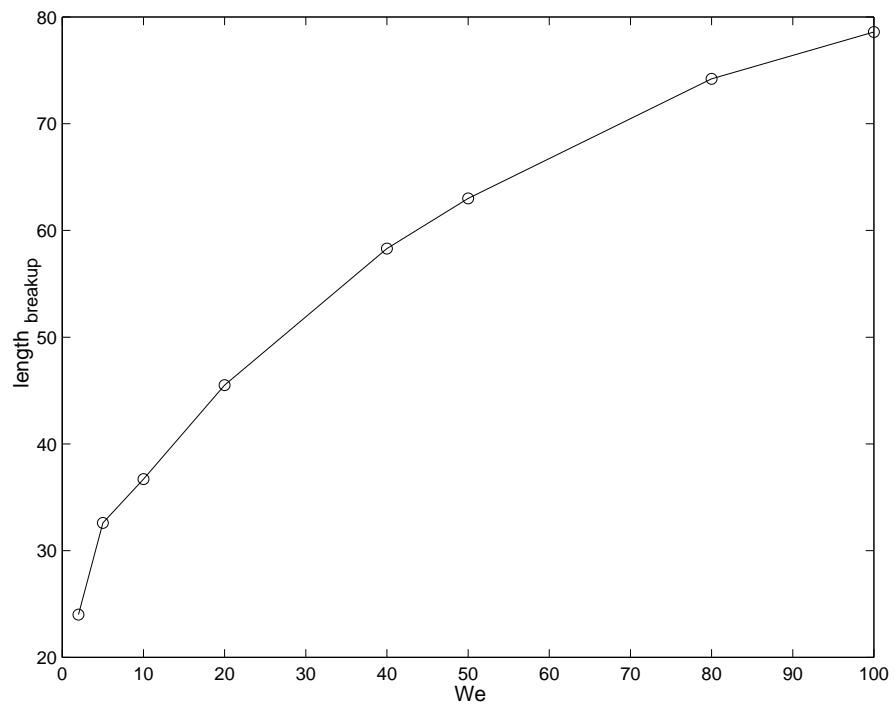

FIG. 9: Parau, Decent, King, Simmons, Wong 


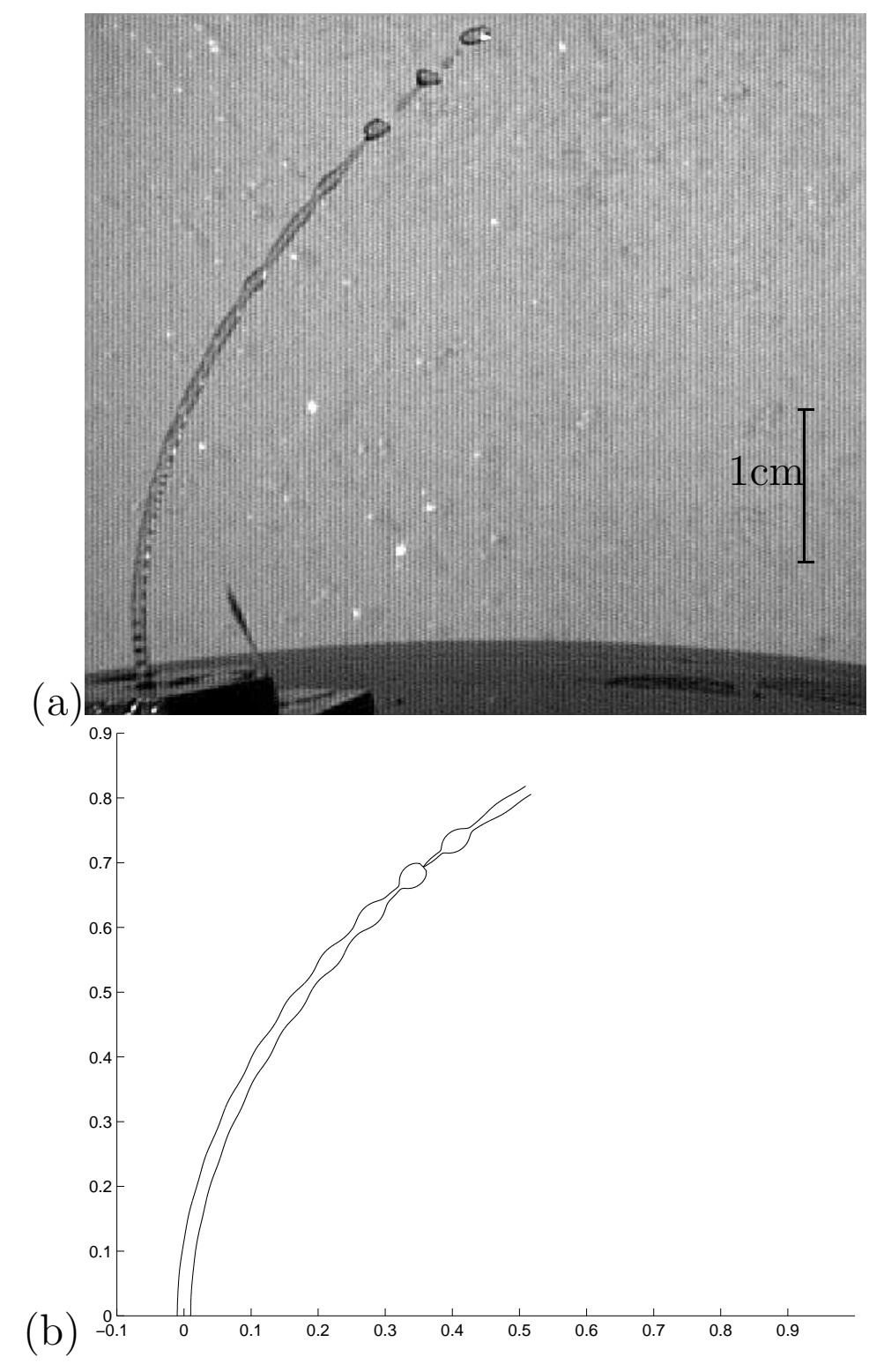

FIG. 10: Parau, Decent, King, Simmons, Wong 


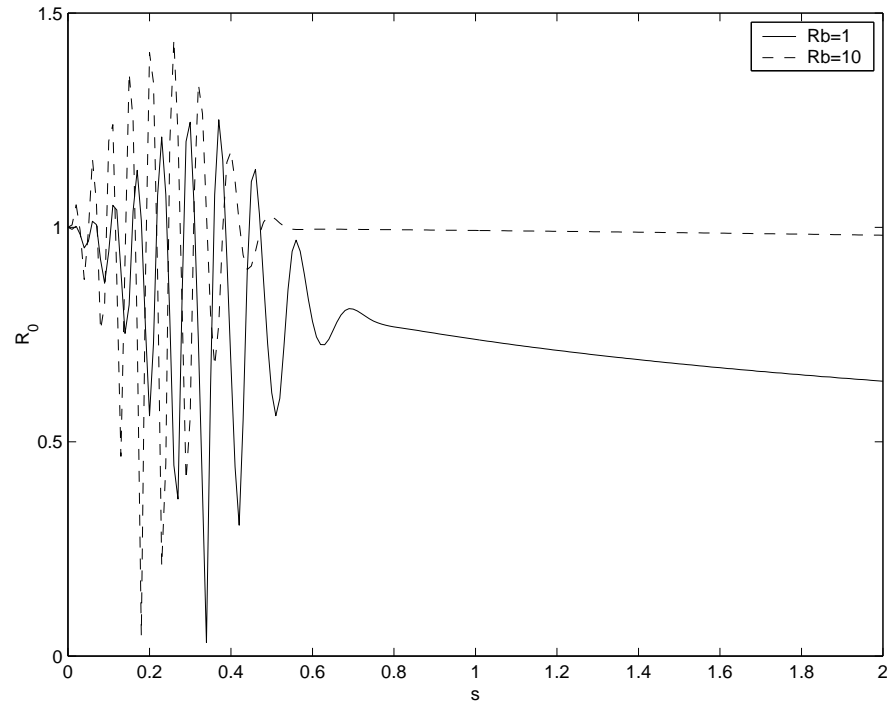

FIG. 11: Parau, Decent, King, Simmons, Wong 


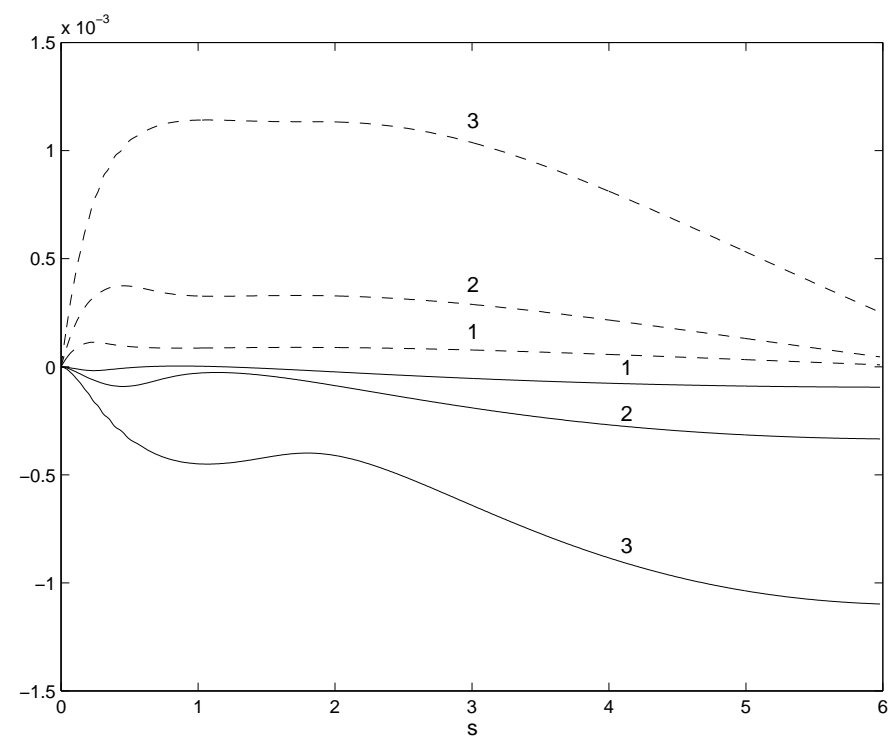

FIG. 12: Parau, Decent, King, Simmons, Wong 


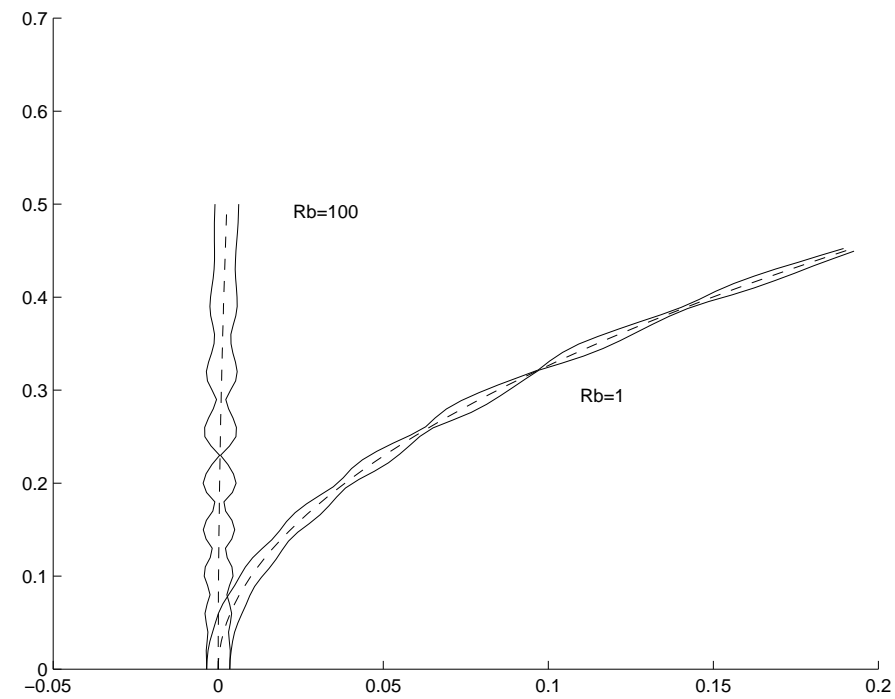

FIG. 13: Parau, Decent, King, Simmons, Wong 